\title{
DIMENSIONING OF FDL BUFFERS FOR OPTICAL BURST SWITCHING NODES *
}

\author{
Christoph M. Gauger \\ Institute of Communication Networks and Computer Engineering (IND) \\ University of Stuttgart, Pfaffenwaldring 47, 70569 Stuttgart, Germany \\ gauger@ind.uni-stuttgart.de
}

\begin{abstract}
Optical burst switching (OBS) is a promising candidate for a more dynamic optical layer in the context of IP over WDM. Although buffering is not mandatory for the functionality of OBS, even simple FDL buffers can improve burst loss probability. In this paper, we first discuss principal buffer architectures and reservation schemes for fiber delay line (FDL) buffers. Then, we investigate key design parameters of FDL buffers like FDL delay, buffer architecture and total number of buffer ports. Finally, we introduce and evaluate strategies for distributing a given number of buffer ports over all FDL's of a feed-forward buffer.
\end{abstract}

Keywords: Optical Burst Switching, WDM, FDL Buffers, Performance Evaluation

\section{Introduction}

IP over WDM is widely seen as the network architecture for the nextgeneration Internet. The intention is to have IP as common platform for a huge variety of applications whereas the optical layer provides sufficient bandwidth and throughput. Regarding the control plane, GMPLS is currently seen as the framework which can provide different levels of IP and WDM integration [1]. The data plane which today relies on statically or dynamically routed wavelengths and provides only this coarse grain bandwidth granularity, will become more and more dynamic as highly variable Internet and transaction traffic increases in volume and importance. OBS [7] and OPS [9] are discussed as candidates for this highly dynamic future optical data plane. While OPS can offer an almost arbitrarily fine granularity, comparable to currently applied electrical packet switching, OBS provides a granularity in between wavelengths

* This work was funded within the TransiNet project (www.transinet.de) by the German Bundesministerium für Bildung und Forschung under contract No. 01AK020C.

A. Bianco et al. (eds.), Next Generation Optical Network Design and Modelling

(C) Springer Science+Business Media New York 2003 


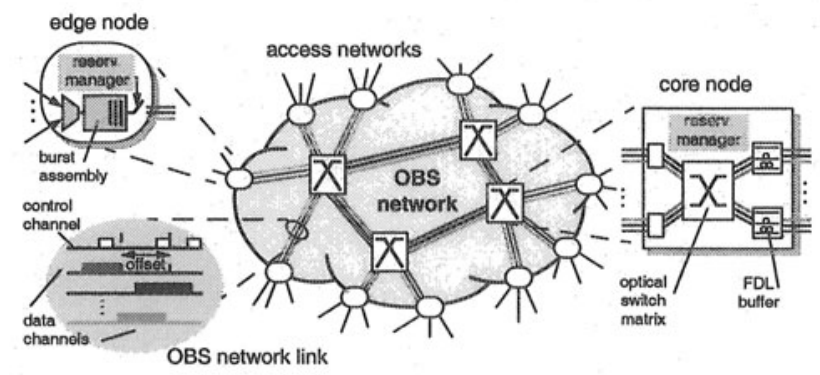

Figure 1. Node and network architecture for optical burst switching

and packets. OBS does neither rely on optical header processing nor on optical buffering. However, in order to further reduce the loss probability of OBS nodes, optical buffers might be deployed solely for contention resolution. Because of relaxed requirements, less complex FDL buffer architectures than the ones proposed in the context of OPS [6] can be employed. This paper studies how FDL buffers for OBS can be dimensioned best.

The remainder of this paper is organized as follows: In Section 2 we give a brief introduction to OBS and the reservation mechanism just enough time (JET). In Section 3 buffer architectures and their key design parameters are discussed and two buffer reservation strategies, PreRes and PostRes, are described. Section 4 evaluates the performance and dimensioning of FDL buffers with respect to several parameters. Finally, Section 5 summarizes our work and addresses further work.

\section{Optical Burst Switching}

\subsection{Definition and Motivation of OBS}

OBS was proposed as a new switching paradigm for optical networks requiring less complex technology than packet switching [7]. The key characteristic of OBS is the hybrid approach, in which header information is signalled out of band and processed electronically while data stays in the optical domain all the time. Also, one-pass, i. e. unacknowledged, reservation and variable length bursts are central to OBS. As OBS is a fast circuit switching (FCS) technique it does not mandate the use of buffers. However, as was shown in $[12,13,11,8,10]$ and is supported by our results presented here and in [4], buffers can improve OBS performance significantly.

As depicted in Figure 1, burst transmission works as follows: $\mathbb{P}$ packets are assembled to data bursts [2] in an OBS edge node. Before transmitting a burst, a reservation request (control packet) is sent on a dedicated channel, e. g. on a separate wavelength. After a basic offset and without waiting for acknowledgement of successful reservations, the data burst is released into the 


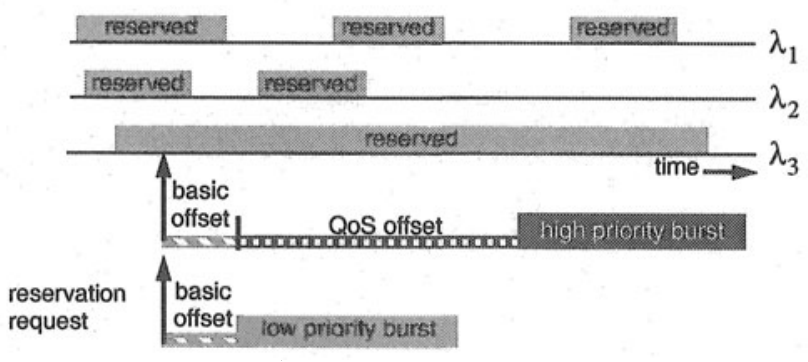

Figure 2. Reservation scenario for bursts of different classes

network. This basic offset has to be large enough to electronically process the control packet and set up the switching matrix in core nodes on the path. When a data burst arrives in a core node the switching matrix has been already set up, i. e. the burst is kept in the optical domain.

\subsection{The JET Reservation Mechanism}

Different mechanisms have been proposed for reservation of wavelengths as well as FDL's for burst transmission. In [3] and [4] we give a detailed overview, classification, and performance comparison of the most important proposals. In JET [12] which is called void filling in [11], predetermined start and end times of each burst transmission are considered for reservation. This allows both efficient utilization of resources and service differentiation. The latter is achieved by assigning an additional quality of service (QoS) offset to a high priority burst which leads to a higher probability of successful reservation which is illustrated in Figure 2 for a scenario with three wavelengths. The low priority burst cannot be served as all wavelengths are already occupied during its transmission time whereas the high priority burst can be served on an available wavelength due to its much larger offset. However, as larger offsets cause additional fixed delay this offset has to be chosen carefully [5].

\section{OBS Nodes with FDL Buffers}

\subsection{FDL Buffer Architectures}

Buffer architectures can be categorized into feed-forward (FF) and feedback (FB) architectures as well as into single-stage and multi-stage structures [6]. In FF buffers data are delayed while forwarded towards the output of the node whereas in FB buffers data are delayed while being fed back to an earlier stage of the node. In single-stage buffers, the delay is realized by a set of fixed-length FDL's while in multi-stage buffers the delay is determined by a cascade of FDL and switch pairs. The capacity of FDL buffers can be increased by using WDM in the FDL's. 

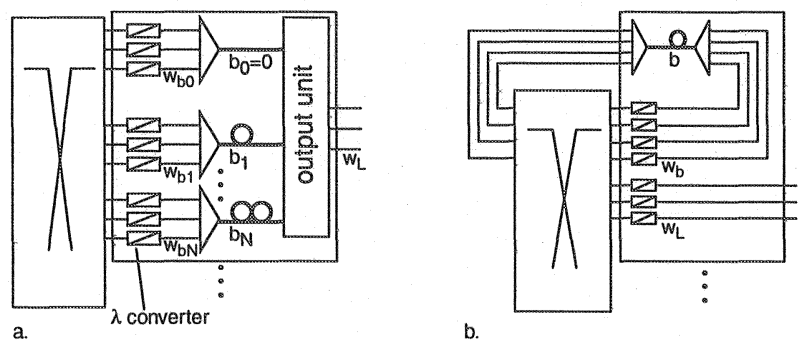

Figure 3. Architecture for a. feed-forward FDL buffer b. feedback FDL buffer

In OBS switches, FDL buffers can be applied as output, input or recirculation buffers and can either be dedicated to a single port or be shared. Figure 3 a shows an FF output buffer and Figure $3 \mathrm{~b}$ an recirculation buffer for one output of an OBS switch. Both are dedicated, single-stage and employ WDM. The FF buffer has a direct line, i.e. delay $b_{0}=0$, with $w_{b 0}$ wavelengths and $N$ FDL's with delays $b_{i}$ and $w_{b i}$ wavelengths, $i=1,2, \ldots, N$. The function of the output unit in Figure 3a depends on the reservation strategies introduced in Section 3.2. The FB buffer comprises a single FDL of delay $b$ and allows a maximum of $Q$ recirculations. There are $w_{L}$ wavelengths on the output fiber and $w_{b}$ in the FDL. In FB buffers, a burst can only reenter the buffer on the same wavelength if its length is shorter than $b$. In case of full wavelength conversion, as assumed here, $b$ does not limit the burst length.

For each wavelength in a buffer FDL there is an input port to the buffer. The total number of buffer input ports, $n_{p}$, equals $\sum_{i} w_{b i}$ in case of FF buffers and $w_{b}$ in case of $\mathrm{FB}$ buffers. The total number of buffer input ports corresponds to the number of additional switch ports needed per output fiber to support an FDL buffer. This directly translates into cost as it determines size of switching matrix or demultiplexer elements as well as number of wavelength converters. For a given total number of buffer ports, assigning these ports to the $N$ FDL's of an FF buffer, i. e. determining all individual $w_{b i}$, is an additional degree of freedom.

Another option in case of FF buffer optimization is choosing individual FDL delays $b_{i}$ such that burst loss probability is minimized. However, in this paper we only consider FF buffers with linearly increasing FDL delays, i. e. $b_{i}=i \cdot b$ for $i=1,2, \ldots, N$.

From a technological point of view, attenuation in $\mathbb{F F}$ and $\mathbb{F B}$ buffers can be compensated by amplifiers dedicated to and exactly tuned to the attenuation of the FDL delay $b_{i}$. In FB buffers, bursts going through the FDL repeatedly accumulate noise, which limits the possible number of recirculations. 
a.

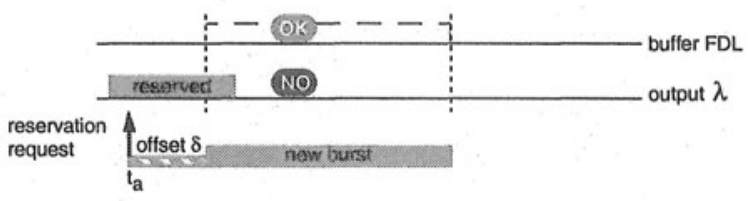

b.

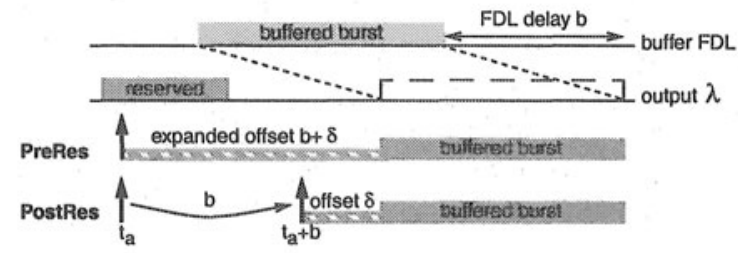

Figure 4. a. Burst blocking event and b. buffer strategies PreRes and PostRes

\subsection{Buffer and Output Channel Reservation}

If a burst cannot make a reservation on an output wavelength in an OBS node with FDL buffers (Figure 3a and 3b) it seeks reservation of a buffer FDL instead. For a system with one output wavelength and one buffer FDL, Figure 4a depicts the arrival of a reservation request at time $t_{a}$ and of the corresponding burst separated by an offset $\delta$. The new burst is blocked from direct transmission by an already reserved burst. However, as a buffer is available during the transmission time of the new burst it can be buffered in the FDL. There are different options for the order and exact time of the FDL and output channel reservation. Figure $4 \mathrm{~b}$ illustrates two possible scenarios in which the new burst buffered in Figure 4a seeks reservation for the time when it leaves the buffer after FDL delay $b$.

So far, the following reservation strategy has been proposed and evaluated [13, 11, 8]: At time $t_{a}$, i. e. when the reservation request of a burst is blocked for the first time, both the shortest available FDL and an output channel are reserved using JET. If either no output channel or no FDL is available for the burst, the burst is discarded. As the output is reserved prior to entering the buffer we call this PreRes. By requesting a wavelength reservation at time $t_{a}$, i. e. with an expanded offset $b+\delta$ prior to the burst transmission (Figure $4 b$ ), this request is prioritized over unbuffered bursts. Hereby, buffered and unbuffered bursts take up the role of high and low priority bursts in offset-based QoS [12], respectively. The differences are that partitioning into two classes is dynamic based on the current contention situation and that the expanded offset is only effective in this node if the control packet is sent to the next node $\delta$ before the burst. From this similarity, we deduce two potential shortcomings: (i) Offsetbased prioritization leads to a higher loss rate of long low priority bursts [3] which in PreRes translates into a higher probability for long bursts to be blocked and sent to the buffer or even to be lost. (ii) In a scenario with QoS classes and 
FDL buffers using PreRes, prioritization of buffered bursts can interfere with QoS classes as the same mechanism is applied in both cases.

In [4], we proposed and evaluated a different mechanism for reservation of buffer and output channels: If a burst is blocked at time $t_{a}$ the shortest available FDL is reserved using JET but no output is reserved at that time. Only at $t_{a}+b$, i. e. after the burst has entered the FDL and $\delta$ before the burst leaves the buffer, an output reservation is requested. As can be seen from Figure $4 \mathrm{~b}$, the offset $\delta$ of the burst stays unaltered for the buffered burst, i. e. it has no priority over newly arriving bursts. We call this mechanism PostRes as output reservation takes place after the burst entered the FDL. In PostRes, all blocked bursts are buffered if buffer space is available. Those unable to reserve an output channel when they leave the FDL are either sent back to the buffer and delayed in case of FB buffers or discarded in case of FF buffers.

The output unit in Figure 3 a consists of wavelength converters as well as of a combiner in case of PreRes and components for selecting bursts which can be transmitted in case of PostRes.

\section{Performance Evaluation}

\subsection{Simulation Scenario}

For an isolated OBS node with a dedicated output buffer comprising either a single FDL FF, a multi-FDL FF buffer or an FB buffer (Figure 3) and employing either PreRes or PostRes reservation strategy, we evaluate the impact of key design parameters on performance. In all simulations, we assume $w_{L}=8$ wavelengths per output fiber and except for Figure 5 a load of 0.8 per output wavelength. This combination of relatively high load and only few wavelengths yields rather high losses. However, this allows us to study the principal behavior of an OBS node in a situation in which FDL buffers are essential. In principle, high losses can also be reduced by increasing the number of wavelengths, e. g. to 64 .

Bursts are generated according to a Poisson process. Burst length is assumed to be negative exponentially distributed with mean $100 \mathrm{kbits}$. This leads to a mean transmission time $h=10 \mu \mathrm{s}$ on a $10 \mathrm{Gbps}$ line. The impact of different transmission time distributions with respect to offset-based QoS is described in [5]. Destination of bursts are uniformly distributed over all output ports. JET is used for wavelength and FDL buffer reservation. Except for Figure 6, all bursts belong to the same class. We use burst loss probability, $P_{\text {loss }}$, as the key performance metric but also discuss transfer time and consider technological constraints and cost. 


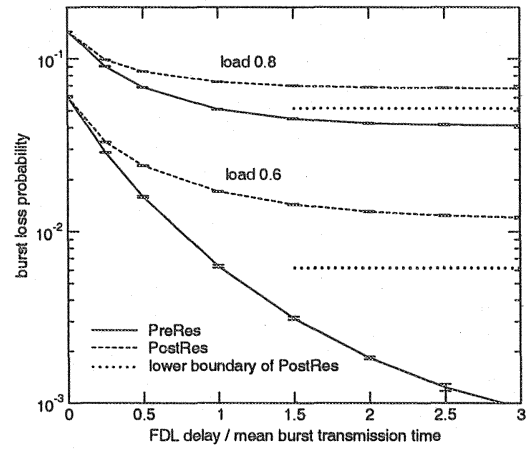

Figure 5. Loss over FDL delay for single FDL feed-forward buffer

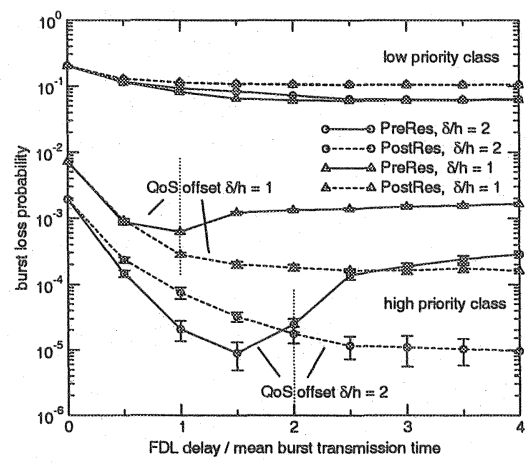

Figure 6. Loss of high priority class over FDL delay $(\delta / h=1$ and $\delta / h=2)$

\subsection{Single FDL Feed-Forward Buffer}

In order to explain the fundamental differences between PreRes and PostRes, we first look at their performance for a single FDL FF buffer without capacity restrictions. Figure 5 shows the burst loss probability for both strategies at load 0.6 and 0.8 over the FDL delay $b$ normalized by the mean burst transmission time $h$. It can be seen that even a single FDL of length $b=3 h$ (which corresponds to $6 \mathrm{~km}$ of fiber) can lower burst loss probability, $P_{\text {loss, }}$, efficiently. For all FDL delays, PreRes outperforms PostRes, whereas the difference is greater for load 0.6. Curves flatten for larger $b$, which shows, that the positive effect of an FDL is limited to the resolution of temporary congestion. As PreRes prioritizes the reservation request of a buffered burst over the one of a newly arriving burst, buffered bursts only compete with long bursts already reserved as well as with requests of other buffered bursts (Section 3.2). Again, this is similar to the JET reservation process with offset-based QoS. However, our analysis from [3] for the burst loss probability of two QoS classes is not applicable here as the partitioning into two classes is not static but dynamic based on the current contention situation. Still, this interpretation tells us that the competition with long active bursts decreases with increasing delay, $b$, which leads to smaller losses.

For PostRes, a lower boundary can be obtained by modeling the initial arrival and the arrival from the buffer as independent and taking into account the load of buffered bursts (repeated call attempt model). Comparing the curves of this lower boundary and PostRes shows that an FDL of limited delay cannot achieve the same performance as a system in which buffers provide arbitrary and almost unlimited delay as assumed for the boundary.

Although PreRes only stores bursts which will be transmitted later it sends more bursts through the buffer than PostRes which stores all blocked bursts 
based on availability. This can be explained by the fact that during a contention situation in PreRes, blocked bursts enter the buffer and reserve the output in advance, which leads to a fragmentation of the output channel. Newly arriving bursts can only reserve an output directly if they fit into a gap formed by active bursts and reservations of buffered bursts [4]. The fact that a substantial share of all bursts is sent through the buffer in PreRes leads to an increased mean transfer time compared to PostRes. As FDL delay is in the order of burst transmission times, i. e. a few microseconds based on above described assumptions, FDL delay can be neglected compared to propagation delays.

As the offset-based QoS differentiation and PreRes contention resolution strategy are based on similar mechanisms an undesired interaction has to be avoided by carefully choosing the respective parameters. Therefore, we study the impact of FDL buffer delay in an OBS node with a single FDL and two service classes. The high priority class has a share of $30 \%$. Figure 6 depicts the burst loss probability over the FDL delay for a QoS offset of one respectively two mean burst transmission times at load 0.8. As already indicated in Section 3.2, it can be seen that for PreRes an FDL that is longer than the QoS offset leads to a reduced isolation between the classes. Furthermore, most of the improvement due to buffering disappears for the high priority class if QoS offset and FDL delay are chosen improperly. Thus, in PreRes, QoS offset and FDL delay have to be adapted such that the QoS offset is always greater than the FDL delay in order to avoid an inefficient use of the system. However, this results in either large QoS offsets, which yield longer delays for all high priority traffic, or restrictions in maximum buffer delay and thus restrictions in the choice of possible buffer architectures.

In contrast, QoS offset and maximum FDL delay can be chosen independently for the PostRes mechanism. Hence, it is possible to have long FDL's (multi-FDL buffers) in order to overcome contention and at the same time have a small QoS offset. For the scenarios in Figure 6, PostRes yields an even better loss probability of the high priority class than PreRes for long FDL's (at the cost of slightly higher losses of the low priority class). In the following, we concentrate on a scenario with a single service class. However, the former results for two service classes have to be considered and principal relations also apply if more complex buffers are used with two service classes.

\subsection{Impact of architecture and FDL delay}

Burst loss probability can be further reduced by FDL buffer architectures which provide more diversity with respect to FDL delay, i. e. with respect to reentry times. For PostRes, an output channel is only reserved when the burst leaves the buffer. If this output channel reservation is blocked, the burst could be sent back to an FDL and seek reservation later in case of an FB buffer but would 


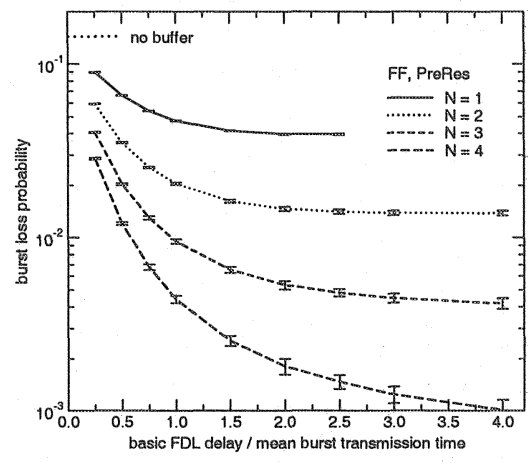

Figure 7. Loss over FDL delay in case of FF buffers with $N=1,2,3,4$

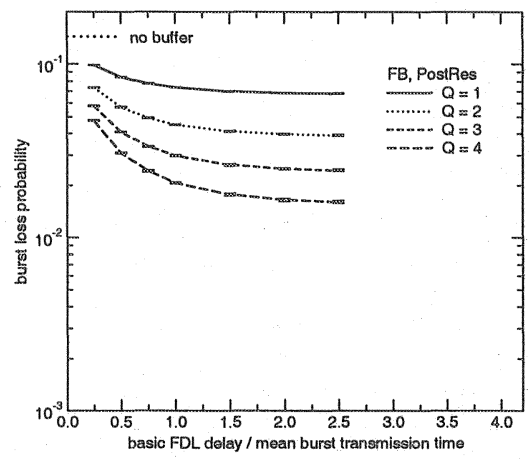

Figure 8. Loss over FDL delay for an FB buffer with $Q=1,2,3,4$

be lost in case of an FF buffer. Also, as PostRes always selects the shortest available FDL multi-FDL FF buffers cannot be used efficiently. In contrast, as PreRes reserves an FDL and an output channel before entering the buffer it cannot exploit the greater flexibility of FB buffers. Therefore, we restrict our investigation to FF buffers with $N=1,2,3,4$ FDL's and linearly increasing delay $b_{i}=i \cdot b(i=1, \ldots, N)$ employing PreRes and to FB buffers with FDL delay $b$ and maximum number of recirculations $Q=1,2,3,4$ employing PostRes.

Figure 7 and Figure 8 depict the burst loss probability $P_{\text {loss }}$ over the basic FDL delay $b$. In both scenarios, the number of wavelengths per FDL is chosen to be no restricting factor. These figures illustrate the impact of FDL delay as well as of number of FDL's, $N$, and maximum number of recirculations, $Q$. In case of the FF buffer with PreRes, it can be seen that introducing more FDL's, i. e. increasing $N$, leads to significantly reduced $P_{\text {loss }}$. In case of FB buffers with PostRes, increasing the maximum number of recirculations, $Q$, also leads to a lower $P_{\text {loss }}$ but the improvement is smaller compared to $F F$ buffers.

In both scenarios, this improvement comes at the cost of increased transfer times-even for constant $b$-as several bursts are buffered in longer FDL's. However, increasing $N$ in FF buffers means additional FDL's including switch ports and amplifier equipment while increasing $Q$ in $\mathrm{FB}$ buffers means few additional switch ports but increased requirements with respect to power and noise budget. In Section 4.4, we study these trade-offs with respect to the number of switch ports as we do not consider the total length of FDL fiber the restricting cost factor.

In case of FF buffers with PreRes and FB buffers with PostRes, burst loss probability always decreases for increasing FDL delays $b$ until a boundary is reached. At load of 0.8 , in case of FF buffers with $N=1$ and $N=2$ curves flatten from approximately $b=2 h$ on while for $N>2$ this boundary is reached 
only for higher values of $b$. In case of FB buffers and all values of $Q$ the boundary is reached at approximately $b=2 h$. In case of lower loads, it can be concluded from Figure 5 that a boundary is only reached for higher values of the base delay.

Summarizing, choosing the basic delay $b$ in the range of a few mean burst transmission times yields significantly improved performance while the fiber delay of the longest fiber in the FF buffer is still in a feasible range. The latter conclusion is valid for burst transmission times up to a few 10's of microseconds as assumed here, however, burst transmission times of milliseconds would lead to infeasibly long FDL's.

In the following, a base delay $b=2 h$ is assumed as it provides an optimal or close to optimal $P_{\text {loss }}$ for most architectures considered.

\subsection{Impact of total number of FDL buffer ports}

So far, the number of wavelengths in an FDL which corresponds to the number of bursts which can be stored simultaneously on the same FDL as well as to the number of switch ports assigned to an FDL has not been limited. As the size of the switching matrix is a key design parameter, we study the impact of the total number of buffer ports, $n_{p}$, on $P_{\text {loss }}$ for an output with $w_{L}=8$ wavelengths. In case of an FF buffer we assume for now that each FDL is assigned an equal number of ports $w_{b i}=w_{b}, i=1,2, \ldots, N$, which results in $n_{p}=N \cdot w_{b}$ ports.

Figure 9 and Figure 10 depict $P_{\text {loss }}$ over the total number of buffer ports, $n_{p}$, for the FF buffer with PreRes and $N=1,2,3,4$ as well as for the FB buffer with PostRes and $Q=1,2,3,4$. For all buffer architectures, $P_{\text {loss }}$ decreases with increasing $n_{p}$-in case of the FF buffer until a bend at $n_{p}=N \cdot w_{L}$ is reached and in case of the FB buffer until a lower boundary is reached for $n_{p}<Q \cdot w_{L}$ ports. Due to the economies of scale relatively fewer ports are needed when increasing $Q$ for reaching the lower boundary. The bend in case of FF with PreRes can be explained by looking at an individual buffer FDL: buffering more than $w_{L}$ bursts at the same time in the same FDL cannot lead to a lower $P_{\text {loss }}$ as only a maximum of $w_{L}$ bursts can leave the buffer at the same time and be sent to the output channels, i. e. only $w_{b} \leq w_{L}$ is a reasonable dimensioning for an individual FDL. This holds true for each FDL in an FF buffer, which leads to the bends at $n_{p}=N \cdot w_{L}$.

For the FF buffer and a given number of buffer ports, $n_{p}$, having a greater number of FDL's, $N$, with a smaller $w_{b}$ yields better loss performance than fewer FDL's with a larger $w_{b}$. However, the latter can be achieved at a lower cost. As the difference is slight for small $n_{p}$ and more distinct for larger $n_{p}$ a small number of FDL's is beneficial if only a small total number of ports, $n_{p}$, is available in order to minimize cost. 


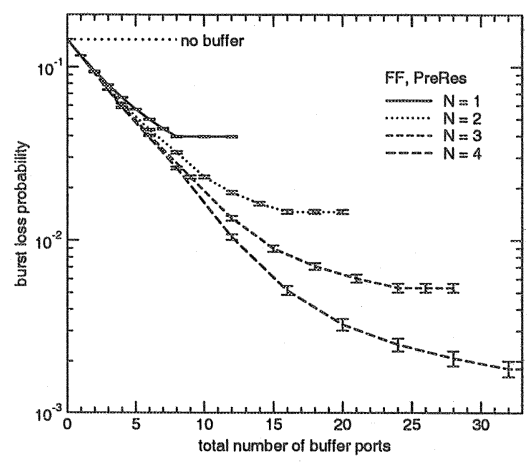

Figure 9. Loss over total number of ports for FF buffers, $N=1,2,3,4$

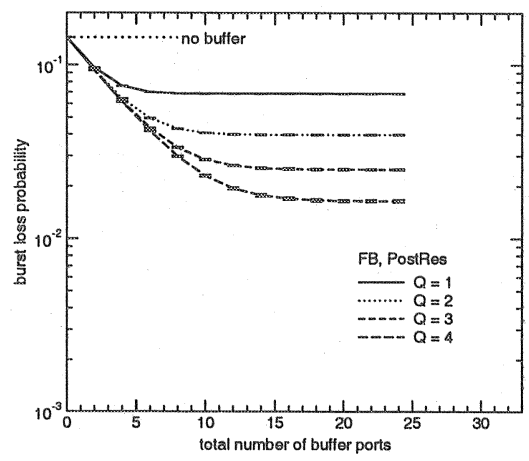

Figure 10. Loss over total number of ports for FB buffers, $Q=1,2,3,4$

Comparing a single FDL FF buffer and an FB buffer with $Q=1,2, \ldots$ under the assumption of an equal number of ports, $n_{p}$, is especially interesting as both scenarios are based on an identical FDL buffer. From Figure 9 and Figure 10 it can be seen that $Q>2$ recirculations in the FB buffer are needed in order to achieve a lower $P_{\text {loss }}$ than the single FDL FF buffer. This is independent of $n_{p}$, however, the difference is marginal for very small $n_{p}$ but increases significantly for larger $n_{p}$. From the technological point of view a key difference of both scenarios is that in an FB buffer bursts traverse a greater number of switching and amplifier elements and therefore accumulate noise. Thus, if only few ports are available an FF buffer is more desirable due to the reduced complexity.

\subsection{Impact of Port Assigmment in FT Buffers}

The impact of the total number of buffer ports, $n_{p}$, of an FF buffer has been investigated under the assumption that all FDL's are assigned an equal number of ports $w_{b i}=w_{b}=n_{p} / N$. The latter assumption raises two questions: (i) Is this assignment strategy optimal? (ii) How should a given number of ports, $n_{p}$, be assigned to $N$ FDL's if $n_{p}$ is not divisible by $N$ ? Regarding the first question, assigning an equal number of ports to each FDL might be beneficial as reentry times of blocked bursts are spread more uniformly over time. However, assigning more ports to shorter FDL's may be advantageous as simulation results show that the mean FDL occupation is higher for the shorter FDL's in a multi-FDL FF buffer. This is due to the fact, that PreRes seeks reservation on the shortest FDL first and only if no reservation is possible it probes the next longer FDL.

In order to find strategies for assignment of FDL ports, we first evaluate the loss probability for all possible port assignments in a two FDL FF buffer with a constant total number of buffer ports $n_{p}$. Each combination of ports for 


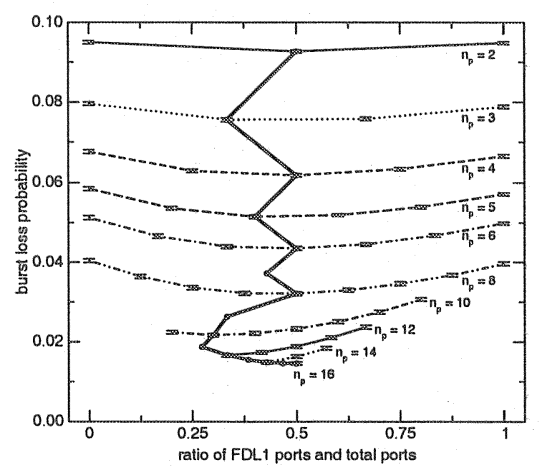

Figure 11. Comparison of combinations of $w_{b 1}$ and $w_{b 2}$ for constant $n_{p}$

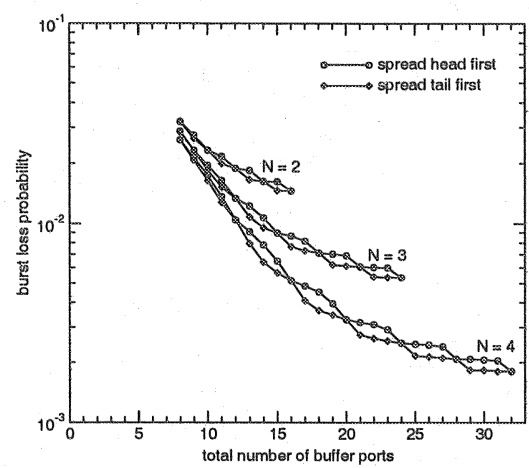

Figure 12. Comparison of spread algorithms

FDL 1 and FDL $2,\left(w_{b 1}, w_{b 2}\right)$, can be characterized by the ratio $w_{b 1} / n_{p}$ of ports assigned to the shorter FDL $w_{b 1}$ and the total number of ports $n_{p}=w_{b 1}+w_{b 2}$. In Figure 11, $P_{\text {loss }}$ is depicted on a linear axis over this ratio $w_{b 1} / n_{p}$ for several values of $n_{p}$ ranging from 2 to 16 . As described in the previous section, assigning more than eight ports to any of the FDL's is not reasonable and is thus not considered here. The fat line in Figure 11 connects the minima of all curves of constant $n_{p}$, even for those curves that have been left out in order to improve clarity of the graph.

For only a few ports, $n_{p}$, the curves are rather flat, symmetric with respect to $w_{b 1} / n_{p}$ and have their minimum either if half of the ports are assigned to the shorter FDL and half to the longer FDL, or in case of an odd $n_{p}$ if the longer FDL is assigned one more port. For a greater port count, $n_{p}$, the curves are no longer symmetric and minima are taken if the longer FDL is assigned the maximum port count of 8 and the shorter FDL is assigned all remaining ports. From the boundary found in Figure 7 for increasing FDL delay as well as from the fact that $P_{\text {loss }}$ is almost identical for the combinations $\left(w_{b 1}=8, w_{b 2}=0\right)$ and $\left(w_{b 1}=0, w_{b 2}=8\right)$ it can be concluded that the different FDL delays are not the main origin of variations of $P_{\text {loss }}$ over $w_{b 1} / n_{p}$ but the different distribution of burst reentry times is. This is also supported by simulations for $b=4 h$ which showed almost identical loss probabilities and minima as depicted in Figure 11 for $b=2 h$.

For two FDL's we conclude that assigning half of the available ports to both FDL's yields best results for a small port count while assigning more ports to the longer FDL is beneficial for a larger port count. 


\subsection{Port Assignment Strategies in FF Buffers}

Based on the findings of the previous section, we introduce and evaluate four special strategies for assigning $n_{p}$ ports to $N$ FDL's. From the results of our evaluations for two FDL's, it can be deduced that depending on the total number of available ports, $n_{p}$, strategies that spread ports over FDL's uniformly or strategies that concentrate ports at either short or long FDL's lead to better loss performance. This categorization motivates the following four strategies:

in spread head (tail) first: The ports are assigned to FDL's one at a time based on a round robin scheme, starting with the shortest (longest) FDL until all ports are assigned.

- concentrate head (tail) with parameter $w_{\max } \leq w_{L}: w_{\max }$ ports are assigned to the $N-1$ shortest (longest) FDL's each and all remaining ports are assigned to the longest (shortest) FDL.

Reasonable domains for the total number of ports, $n_{p}$, in the strategies spread and concentrate are $N \leq n_{p} \leq N \cdot w_{L}$ and $(N-1) w_{\max }<n_{p} \leq N \cdot w_{\max }$, respectively. The lower limits account for the fact that there has to be at least one port per FDL and the upper limit accounts for the fact that assigning more than $w_{L}$ ports to an FDL does not improve performance or that at most $w_{\max }$ are allowed.

Figure 12 compares the burst loss probability of the strategies spread head first and spread tail first for a given number of ports, $n_{p}$, and $N=2,3,4$. It can be seen that the curves of both strategies are very close together, with the tail first strategy always slightly better except for the points in which both strategies produce the same assignment already studied in Figure 9. However, for some medium values of $n_{p}$ the improvement of tail first is about as big as the improvement achieved by adding an additional FDL but comes at much lower cost. As both spread strategies have almost identical mean transfer times the tail first strategy is more suitable than head first.

The strategies concentrate depend on an additional parameter, $w_{\max }$, and depending on that parameter are defined on a smaller domain than spread. Figure 13 depicts the burst loss probability for the strategies concentrate and two values of $w_{\max }$. For $w_{\max }=8$, the concentrate tail leads to significantly lower $P_{\text {loss }}$ for all $N$ except for the points in which both strategies produce the same assignment. While $P_{\text {loss }}$ decreases rather uniformly in case of concentrate head, it drops more radically with concentrate tail for assignments in which there are only a few ports in the shortest FDL. For $w_{\max }=4$, the difference is much smaller or even disappears. Thus, Figure 13 supports findings from Figure 11: For a small $n_{p}$ concentrate head and tail are almost identical while for a larger $n_{p}$ concentrate tail definitely has a lower $P_{\text {loss }}$. 


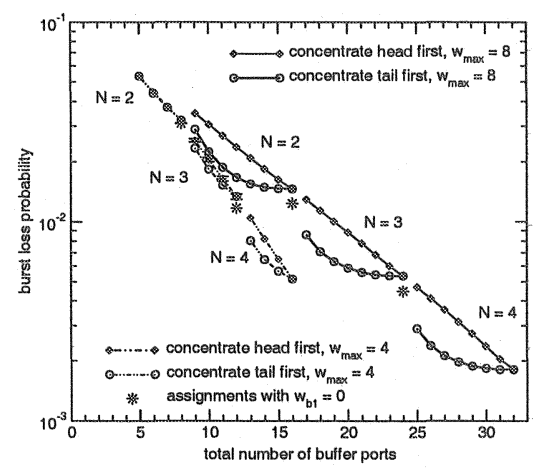

Figure 13. Comparison of concentrate algorithms for $w_{\max }=4$ and 8

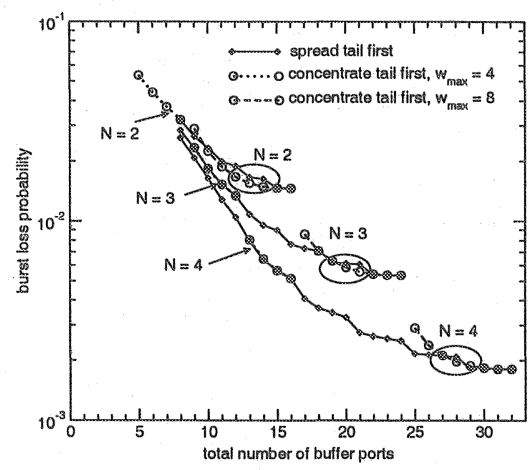

Figure 14. Comparison of spread and concentrate algorithms

When concentrate tail is used and all $N$ FDL's in a buffer are assigned $w_{\max }$ ports adding one more port means adding an additional longer FDL, shifting all ports assigned so far to the $N-1$ longest FDL's and assigning the new port to the shortest FDL. Thereby, the mean FDL delay is increased by approximately $b$ which has some positive impact on losses (Figure 7). In order to quantify this impact, Figure 13 also contains values of $P_{\text {loss }}$ (star symbols) for the case in which the shortest FDL is assigned no port but the $N-1$ longer FDL are assigned $w_{\max }$ ports each, e.g. for $N=4$ and $n_{p}=24$ ports. Comparing these values with $P_{\text {loss }}$ obtained for the same $n_{p}$ but $N=3$, it can be seen that the effect of adding one more port dominates the effect of the increased mean FDL delay.

In Figure 14, we finally compare spread tail first and concentrate tail which performed best so far. For $N>2$ concentrate tail and spread tail first perform almost equally for both values of $w_{\max }$. However, for $N=2$ concentrate tail achieves lower losses for several values which has been discussed in Figure 11.

Concluding, we found that in case of $N>2$ the spread tail first strategy produced the lowest loss probability for by far most port counts, $n_{p}$, and should therefore be used to assign ports in the process of FDL buffer dimensioning. In the case of only two FDL's, concentrate tail is more advantageous.

\section{Conclusions and Outlook}

In this paper, dimensioning of feed-forward as well as feedback FDL buffer architectures for OBS networks has been investigated considering two reservation strategies for FDL buffers, PreRes and PostRes.

The impact of key design parameters such as FDL delay, buffer architecture, total number of buffer ports as well as assignment of buffer ports to individual FDL's on burst loss probability has been studied. For the assignment of buffer 
ports to FDL's in multi-FDL feed-forward buffers four strategies have been introduced and compared.

We demonstrated that FDL delays in the range of a few mean burst transmission times yield close to optimal performance for all architectures and reservation strategies at high load. Increasing the number of FDL's in feed-forward buffers or the number of recirculations in feedback buffers has significant impact for a large total number of buffer ports but leads to only minor improvements for a small number of ports. Assigning a given number of buffer ports to the FDL's of a feed-forward buffer based on the introduced strategy spread tail first, which spreads ports over all FDL's uniformly starting with the longest FDL, yielded lowest burst loss probability for more than two FDL's.

Further work could include evaluation of feed-forward buffers in which the delays of the FDL's are no integer multiples of the basic delay. Also, comparison of architectures and dimensioning of FDL buffers could include technological constraints of special node architectures, e. g. employing semiconductor optical amplifiers. Finally, adapted reservation strategies for two service classes as well as only limited wavelength conversion capability in the node could be investigated.

\section{Acknowledgments}

The author would like to express his gratitude to K. Dolzer and S. Bodamer for invaluable discussions and to M. Scharf for his work on the simulation tool. The author would also like to thank H. Franz-Feng, E. Patzak, and J. Saniter for their kind support regarding technology issues as well as the reviewers for their comments.

\section{References}

[1] A. Banerjee, J. Drake, J. P. Lang, B. Turner, K. Kompella, and Y. Rekhter. Generalized multiprotocol label switching: an overview of routing and management enhancements. IEEE Communications Magazine, 39(1):144-150, January 2001.

[2] K. Dolzer and C. Gauger. On burst assembly in optical burst switching networks-a performance evaluation of just-enough-time. In 17th International Teletraffic Congress (ITC 17), pages 149-160, Salvador, Brazil, December 2001.

[3] K. Dolzer, C. Gauger, J. Späth, and S. Bodamer. Evaluation of reservation mechanisms for optical burst switching. AEÜ International Journal of Electronics and Communications, 55(1):18-26, January 2001.

[4] C. Gauger, K. Dolzer, and M. Scharf. Reservation strategies for FDL buffers in OBS networks. Technical Report 40, Institute of Communication Networks and Computer Engineering, University of Stuttgart, November 2001.

[5] C. Gauger, K. Dolzer, J. Späth, and S. Bodamer. Service differentiation in optical burst switching networks. In Proceedings of the 2. ITG Symposium on Photonic Networks, pages 124-132, Dresden, March 2001. 
[6] D. K. Hunter, M. C. Chia, and I. Andonovic. Buffering in optical packet switches. IEEE Journal on Lightwave Technology, 16(12):2081-2094, December 1998.

[7] C. Qiao and M. Yoo. Optical burst switching (OBS)-a new paradigm for an optical Internet. Journal of High Speed Networks, 8(1):69-84, January 1999.

[8] L. Tancevski, S. Yegnanarayanan, G. Castanon, L. Tamil, F. Masetti, and T. McDermott. Optical routing of asynchronous, variable length packets. IEEE Journal of Selected Areas in Communications, 18(10):2084-2093, October 2000.

[9] R. S. Tucker and W. D. Zhong. Photonic packet switching: an overview. IEICE Transactions on Communications, E52-B(2):254-264, February 1999.

[10] J. S. Turner. Terabit burst switching. Journal of High Speed Networks, 8(1):3-16, January 1999.

[11] Y. Xiong, M. Vanderhoute, and C. C. Cankaya. Control architecture in optical burst-switched WDM networks. IEEE Journal of Selected Areas in Communications, 18(10):1838-1851, October 2000.

[12] M. Yoo, M. Jeong, and C. Qiao. A high speed protocol for bursty traffic in optical networks. In 3rd SPIE Conference on All-Optical Communication Systems, pages 79-90, Dallas, November 1997.

[13] M. Yoo and C. Qiao. QoS performance in IP over WDM networks. IEEE Journal of Selected Areas in Communications, 18(10):2062-2071, October 2000. 